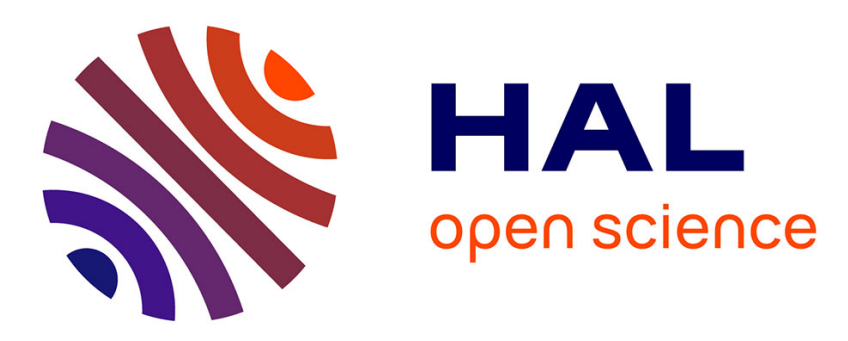

\title{
Experimental characterization of the noise generated by an airfoil oscillating above stall
}

\author{
David Raus, Lisa Sicard, Benjamin Cotté, Romain Monchaux, Emmanuel \\ Jondeau, Pascal Souchotte, Michel Roger
}

\section{- To cite this version:}

David Raus, Lisa Sicard, Benjamin Cotté, Romain Monchaux, Emmanuel Jondeau, et al.. Experimental characterization of the noise generated by an airfoil oscillating above stall. AIAA AVIATION 2021 FORUM, Aug 2021, VIRTUAL EVENT, United States. 10.2514/6.2021-2291 . hal-03342329

\author{
HAL Id: hal-03342329 \\ https://hal.science/hal-03342329
}

Submitted on 13 Sep 2021

HAL is a multi-disciplinary open access archive for the deposit and dissemination of scientific research documents, whether they are published or not. The documents may come from teaching and research institutions in France or abroad, or from public or private research centers.
L'archive ouverte pluridisciplinaire HAL, est destinée au dépôt et à la diffusion de documents scientifiques de niveau recherche, publiés ou non, émanant des établissements d'enseignement et de recherche français ou étrangers, des laboratoires publics ou privés. 


\title{
Experimental characterization of the noise generated by an airfoil oscillating above stall
}

\author{
David Raus*, Lisa Sicard ${ }^{\dagger}$, Benjamin Cotté ${ }^{\ddagger}$ and Romain Monchaux ${ }^{\S}$. \\ Institute of Mechanical Sciences and Industrial Applications (IMSIA), ENSTA Paris,CNRS, CEA, EDF, Institut \\ Polytechnique de Paris, France \\ Emmanuel Jondeau ${ }^{\mathbb{I}}$, Pascal Souchotte $\|$ and Michel Roger **. \\ Ecole Centrale de Lyon, LMFA, Ecully, 69131, France
}

\begin{abstract}
The present work investigates the noise emitted by an airfoil oscillating above stall, thanks to synchronized surface pressure and far-field acoustic measurements in an anechoic open-jet wind tunnel. Both NACA0012 and a NACA63 418 airfoils are tested in order to investigate the effects of the airfoil shape on the static and dynamic stall noise. For static configurations, a smoother transition to stall noise is obtained for the cambered $\mathrm{NACA63}_{3} 418$ airfoil, with a gradual increase and shift of the noise spectra at low frequencies as the separation point moves closer to the leading edge of the airfoil. Phase-averaged time-frequency analysis of the oscillating airfoil noise reveals that the light-stall noise and deep-stall noise regimes commonly observed for static airfoils also take place during the oscillations. Increasing the frequency of the oscillations leads to an increase of the duration and amplitude of the stall onset broadband noise, and a delay of the dynamic stall noise to greater angles of attack. In the same way, the dynamic stall noise is delayed for the $\mathrm{NACA63}_{3} 418$ compared to the NACA0012, causing the stalled phase to be shorter for the NACA63 ${ }_{3} 418$. For the NACA0012, the noise does not depend on the angle of attack before the onset on stall. For the $\mathrm{NACA63}_{3} 418$, the noise gradually increases in the pre-stall regime and the stall noise is preceded by a high amplitude separation noise.
\end{abstract}

\section{Introduction}

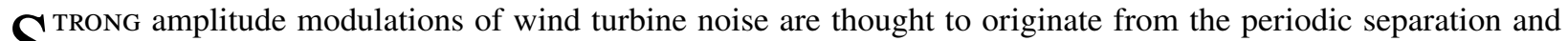

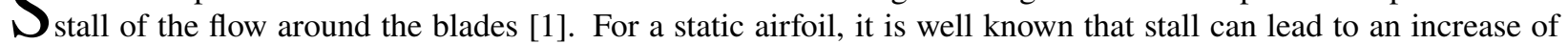
the amplitude of the emitted noise up to $10 \mathrm{~dB}$ at low frequencies compared to the trailing-edge noise of an attached turbulent boundary layer [2]. For a static airfoil at stall, two noise regimes are observed [3, 4]. The light-stall regime is obtained close to the static stall angle and is characterized by a high amplitude broadband low frequency noise. For very large angles of attack, the deep-stall regime exhibits a diminution of the broadband noise amplitude but the presence of a low-frequency narrow-band peak, that can originate from shear layer instabilities and large scale vortex shedding [3]. Stall noise prediction models based on the Curle's analogy have been developped [3, 5], but the mechanisms of generation of the static stall noise are still not understood. Recently, Lacagnina et al. [6] performed combined far-field acoustic, hot-wire and Particle Image Velocimetry (PIV) measurements in order to investigate the mechanisms of noise generation on a NACA65-(12)10 airfoil at high angles of attack. Their results suggest that the stall noise could originate from the interaction between the shear layer (separating the free stream and the separated boundary layer region) and the suction side of the airfoil. Instabilities and coherent structures in the detached shear layer and shear layer flapping could induce unsteady hydrodynamic pressure on the airfoil surface that can be scattered at the trailing edge and radiate to the far-field. Direct Numerical Simulations (DNS) coupled with a Ffowcs Williams and Hawkings solver were used to study the noise emitted by a stalled NACA0012 at low Reynolds number [7]. The simulations confirm that shear layer vortices are convected on the suction side of the airfoil and induce strong pressure fluctuations that are scattered at the trailing

\footnotetext{
*Postdoctoral Researcher, ENSTA Paris, Institute of Mechanical Sciences and Industrial Applications, david.raus@ensta-paris.fr.

${ }^{\dagger}$ PhD student, ENSTA Paris, Institute of Mechanical Sciences and Industrial Applications, lisa.sicard@ensta-paris.fr

† Assistant Professor, ENSTA Paris, Institute of Mechanical Sciences and Industrial Applications, benjamin.cotte@ensta-paris.fr

$\S$ Assiociate Professor, ENSTA Paris, Institute of Mechanical Sciences and Industrial Applications, romain.monchaux@ensta-paris.fr

IIResearch Engineer, Ecole Centrale de Lyon, LMFA, emmanuel.jondeau@ec-lyon.fr

" Research Engineer, Ecole Centrale de Lyon, LMFA, pascal.souchotte@ec-lyon.fr

**Professor, Ecole Centrale de Lyon, LMFA, michel.roger@ec-lyon.fr
} 


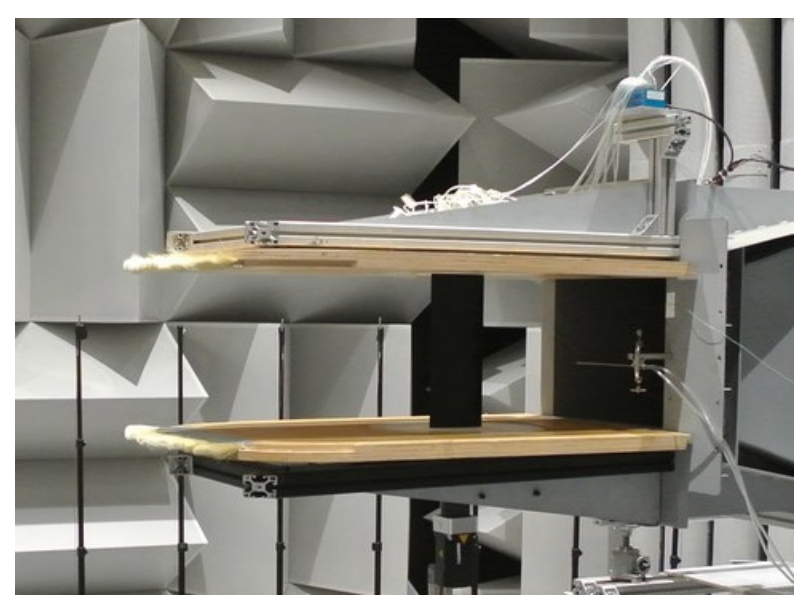

(a)
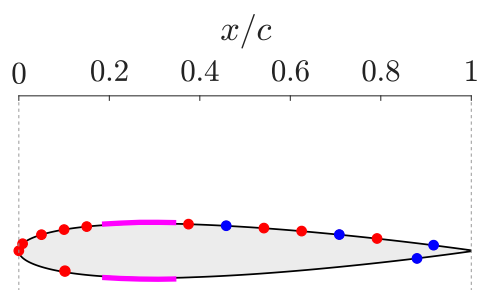

NACA0012

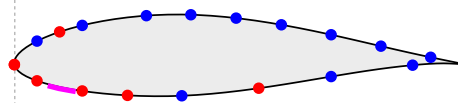

$\mathrm{NACA} 63_{3} 418$

Fig. 1 (a) Picture of the nozzle exit (b) Schematic of the two airfoil shapes and positions of the surface pressure measuring points. Red dots and blue dots show the positions where only the steady-state surface pressure is measured and the positions where both the steady and fluctuating wall pressures are measured, respectively. Purple areas show the positions of the tripping tapes.

edge as a dipole pulse. Experimental data investigating the effect of the camber and thickness of the airfoil on static stall noise is very limited. For the deep-stall regime, Laratro et al. [4] observe that the thickness of the airfoil has a limited effect on its acoustic signature.

Experimental studies of the noise emitted by an oscillating airfoil have recently been performed [8-[11]. Zhou et al. [9] studied the noise emitted by a NACA0012 airfoil at low Reynolds number and oscillating below the stall angle. The authors observe that the laminar boundary layer tonal noise takes place twice per oscillation cycle, once during the upstroke motion of the airfoil and once during the downstroke motion. The angles of attack at which the tonal noise takes place are delayed if the frequency of oscillation increases. The noise emitted by a NACA0012 oscillating above stall was studied in an aeroacoustic wind-tunnel with a Kevlar-walled test section [10]. Time-frequency analysis of the surface pressure fluctuations near the trailing-edge shows that the stalled phase of the pitching motion is associated with an increase of the spectra at low frequencies, that could be attributed to the dynamic stall vortex development. Raus et al. [11] studied the noise emitted by an oscillating NACA0012 in an open-jet wind-tunnel at Reynolds number $R e_{c}=U c / v=2.1 \times 10^{5}$ with $\mathrm{U}, \mathrm{c}$ and $v$ the free-stream velocity, the airfoil chord and the kinematic viscosity of air, respectively. Results show that the light-stall and deep-stall noise regimes also take place during the pitching motion for low oscillation frequencies, and that the amplitude and duration of the broadband noise at stall onset increase as the frequency of the oscillation increases. A delay in the apparition of the stall noise is observed in comparison with static configurations, giving rise to a hysteresis of the stall noise with respect to the angle of attack. These studies did not investigate the effect of the airfoil shape on the emitted noise.

In this paper, the effect of the shape of the airfoil on the dynamic stall noise is studied. Synchronized surface pressure and far-field acoustic measurements are performed with airfoils oscillating above stall at Reynolds number $R e_{c}=4.1 \times 10^{5}$, in order to analyze the variations of the emitted noise during the oscillations. Two airfoil shapes are tested: a NACA0012 and a NACA63 3418 . The NACA6 $3_{3} 418$ is a cambered airfoil that was first designed for use on aircrafts but since then was widely used on wind turbine blades [12, 13]. This airfoil is known to have a high aerodynamic efficiency and to avoid abrupt stall that could lead to vibrations of the wind turbine blade [14]. It is compared to a NACA0012, that was already tested at Reynolds number $R e_{c}=2.1 \times 10^{5}$ in the same experimental setup [11].

\section{Experimental setup}

The experiments were conducted in the anechoic wind tunnel of Ecole Centrale de Lyon (ECL). This wind tunnel consists of an open-jet with a rectangular $0.4 \mathrm{~m} \times 0.3 \mathrm{~m}$ nozzle exit, followed by two horizontal end-plates guiding 


\begin{tabular}{c|c|c|c|c|c|c}
\hline Exp. & Airfoil shape & $\alpha_{0}\left(^{\circ}\right)$ & $\alpha_{1}\left(^{\circ}\right)$ & $f_{o}(\mathrm{~Hz})$ & $k$ & $k_{\star}$ \\
\hline 1 & NACA63 $_{3} 418$ & 15 & 15 & 0.70 & 0.005 & $1.3 \times 10^{-3}$ \\
2 & NACA63 $_{3} 418$ & 15 & 15 & 1.30 & 0.01 & $2.6 \times 10^{-3}$ \\
3 & NACA63 $_{3} 418$ & 15 & 15 & 3.30 & 0.025 & $6.5 \times 10^{-3}$ \\
4 & NACA0012 & 15 & 15 & 1.33 & 0.01 & $2.6 \times 10^{-3}$ \\
5 & NACA0012 & 15 & 15 & 2.65 & 0.02 & $5.2 \times 10^{-3}$ \\
\hline
\end{tabular}

Table 1 Parameters associated with the five oscillating airfoil configurations.

the incoming flow, as shown in Fig. 11a. The test section of the wind tunnel is enclosed in an anechoic chamber of dimensions $8 \mathrm{~m} \times 9 \mathrm{~m} \times 10 \mathrm{~m}$. Two airfoils of chord $c=12 \mathrm{~cm}$ and of span $s=30 \mathrm{~cm}$ were tested: a NACA0012 airfoil and a NACA $633_{3} 418$ airfoil. The airfoils were subjected to a flow of free-stream velocity $U=50 \mathrm{~m} / \mathrm{s}$, corresponding to a Reynolds number $R e_{c}=U c / v=4 \times 10^{5}$. As shown in Fig. $1 \mathrm{p}$, tripping tapes were placed near the leading edge of the airfoils in order to control the developpment of turbulent boundary layers on the airfoil and avoid the generation of the laminar boundary layer tonal noise [2]. For the NACA0012, a tripping tape was placed on both sides of the airfoil between $x / c=0.2$ and $x / c=0.4$ while for the NACA63 3418 , the tape was only placed on the pressure side of the airfoil between $x / c=0.1$ and $x / c=0.15$. These tripping tapes were chosen because they prevent the generation of the tonal noise without heavily modifying the lift curve of the airfoil. Both airfoils were subjected to a sinusoidal pitching motion about their center chord, thanks to a motor placed below the lower end-plate. The sinusoidal motion was implemented as:

$$
\alpha_{d, g}(t)=\alpha_{0}+\alpha_{1} \sin \left(2 \pi f_{0} t\right),
$$

where $\alpha_{d, g}(t)$ is the instantaneous angle of attack of the airfoil, $f_{0}$ is the oscillation frequency and $\alpha_{0}=\alpha_{1}=15^{\circ}$. These angles are the geometric angles of attack imposed by the motor. In an open-jet wind tunnel, the flow deviates from the nozzle axis because of the presence of the airfoil. The effective angles of attack of the airfoil are thus smaller than these geometric values. Incidence corrections suggested by Brooks et al. [15] are commonly used to estimate the effective angle of attack for static airfoils, but have not been validated for an oscillating airfoil. Consequently, in the following, only geometric angles of attack are used. For the NACA63 3418 , three reduced frequencies are tested $k=0.005, k=0.01$ and $k=0.025$ with $k=\pi f_{0} c / U$. For the NACA0012, two reduced frequencies are investigated $k=0.01$ and $k=0.02$. Comparison of the dynamic stall process between the two airfoils is thus possible for $k=0.01$. Parameters associated with the experiments are given in Table 1]. Following Sheng et al. [16] who define the reduced pitch rate as $k_{\star}=\alpha_{1} k$ and estimate the boundary between quasisteady and dynamic stall at $k=0.01$ for NACA airfoils, the configurations studied in the present paper are in the quasisteady regime.

The two airfoils are instrumented with pin-holes located in the mid-span plane, along the chord of the airfoils. These pin-holes are connected by capillary tubes to a Kulite KMPS-1-64 pressure scanner to measure the steady wall pressure at a sampling frequency $f_{s}=1.1 \mathrm{kHz}$. As shown in Fig. 1 $1 \mathrm{p}$, some of the pin-holes are also connected to Brüel \& Kjær 4958 type microphones through a T-junction to acquire the fluctuating wall pressure signals at a sampling frequency $f_{s}=51.2 \mathrm{kHz}$. This Remote-Microphone Probe (RMP) technology and the in-situ calibration of the microphones are described in Reference [17]. The local surface pressure fluctuations measured by the Remote-Microphone Probes are of acoustic and aerodynamic natures. For attached or separated turbulent boundary layers such as the ones studied near the trailing-edge in the present study, the measured surface pressure signals are essentially of hydrodynamic nature as the pressure fluctuations induced by aerodynamic structures have much larger amplitude than the one induced by acoustic processes [17]. The positions of the surface pressure measuring points are shown in Fig. 1p. For the NACA0012, pressure taps are mainly located on one side of the airfoil and the fluctuating surface pressure is only measured close to the airfoil trailing-edge. For the NACA $63_{3} 418$, pressure taps are located on both sides of the airfoil and the fluctuating surface pressure is also measured close to the leading edge.

Far-field noise measurements were performed with a Gras 46BE microphone placed in the mid-span plane, 2 meters away from the airfoil pressure side center-chord, and oriented perpendicular to the incoming flow. In order to characterize the background noise generated by the wind tunnel, far-field noise measurements were also performed in the same wind conditions but without the airfoil in the test section. The sources of the background noise (noise of the jet, trailing-edge noise of the horizontal plates...) are assumed to be identical when the airfoil is added in the test section. Precautions must be taken at very high angles of attack, as the background noise could be slightly modified at low frequencies because of the airfoil [3]. 


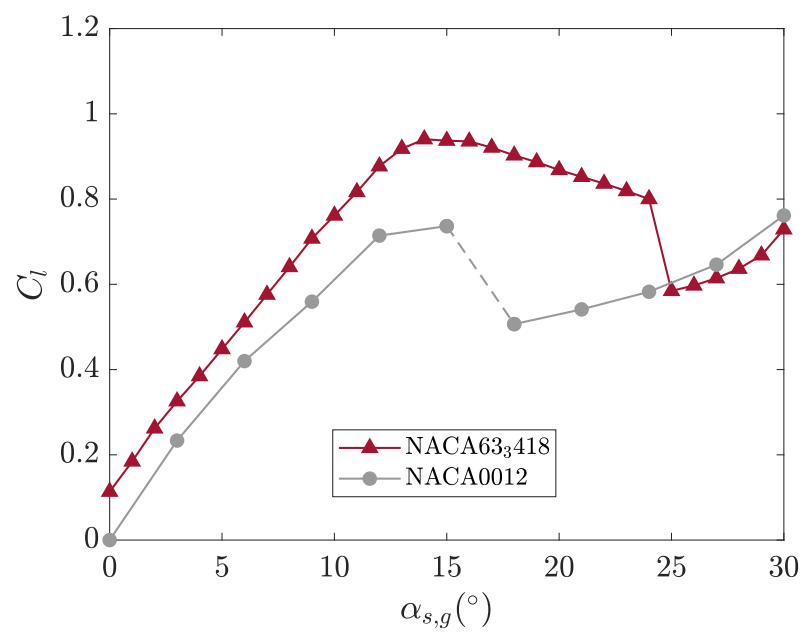

(a)

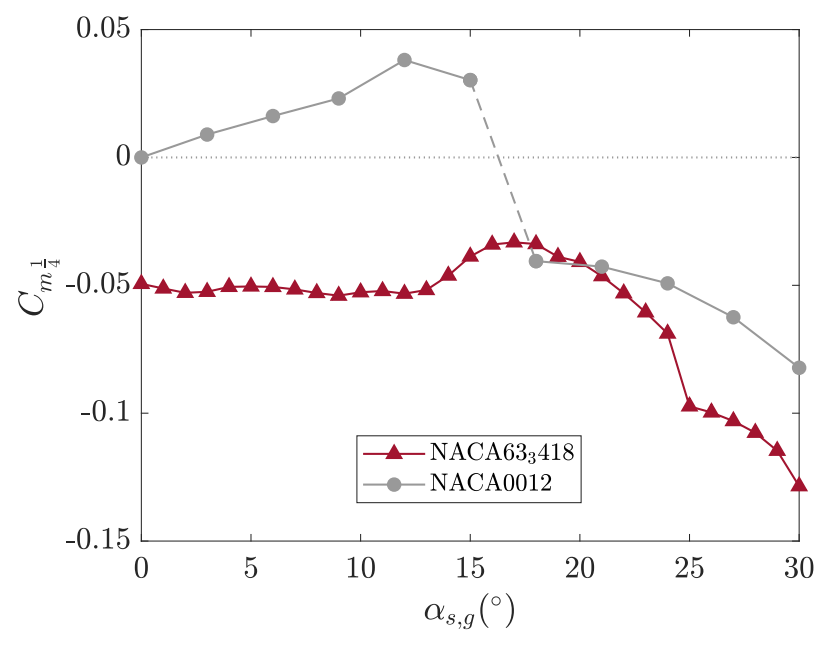

(b)

Fig. 2 Comparison of the (a) lift coefficients and (b) pitching moment coefficients at the quarter chord between the two NACA airfoils. The angle resolution being too poor to measure precisely the static stall angle for the NACA0012 airfoil, dashed lines where choosen around the stall angle in order not to mislead the reader.

\section{Static airfoil}

This section investigates the effects of the airfoil shape on the static stall noise. For static configurations, the surface pressure and the far-field acoustic pressure signals are measured over periods of 30 seconds. The Power Spectral Density (PSD) of the signals is calculated using the Welch's method, with a Hamming window size of 6400 samples and 50\% overlap. The resulting PSD have a frequency resolution of $8 \mathrm{~Hz}$. For the NACA0012 airfoil, a 3 degrees increment was chosen between static angles measurements while for the NACA63 3418 airfoil, a 1 degree increment was chosen.

The lift and pitching moment coefficients are obtained by integrating the static pressure along the chord of the airfoils. The comparison of these coefficients obtained for the two NACA airfoils is presented in Fig. 2 whereas the distribution of static pressure on the suction side of the airfoils is shown in Fig. 3. For small angles of attack, the lift coefficient increases linearly for both airfoils. The pitching moment coefficient at the quarter chord $C_{m_{\frac{1}{4}}}$ is positive and increases with the angle of attack for the NACA0012, whereas it is negative and constant for the NACA63 418 . The lift coefficient stops increasing for $\alpha_{s, g}>15^{\circ}$ for both airfoils, as the flow starts separating on the suction side of the airfoil. A plateau of static pressure is thereby observed on the suction side of the airfoils in Fig. 3. Abrupt stall occurs for the NACA0012 for $15^{\circ}<\alpha_{s, g}<18^{\circ}$. For the NACA $63_{3} 418$, the separation point gradually moves upward and the lift progressively decreases up until $\alpha_{s, g}=24^{\circ}$. Full stall is finally observed for the NACA63 418 at $\alpha_{s, g}=25^{\circ}$, with an abrupt loss of lift. For $\alpha_{s, g}>25^{\circ}$, close lift coefficients are obtained for the two airfoils. For the NACA63 418 , the separation of the flow leads to a slight increase of the pitching moment coefficient, followed by a strong decrease at stall. For the NACA0012, the pitching moment coefficient becomes negative at stall.

PSD of surface pressure fluctuations at $x / c=0.92$ on the suction side of the airfoils are shown in Fig 4 for a selection of static angles of attack. At very low angle of attack, a similar flat surface pressure spectrum is observed for both airfoils. For higher angles of attack, an increase of the spectrum amplitude in the low frequencies is obtained due to the flow separating and the turbulent structures near the trailing edge getting larger. For the NACA0012, the abrupt separation at $\alpha_{s, g}=15^{\circ}$ leads the surface pressure spectrum to rapidly increase and exhibit a power law behavior for $f c / U>0.5$. The transition to a power law behavior is smoother for the NACA63 418 , as the separation point gradually moves upward the airfoil chord. For $\alpha_{s, g}=18^{\circ}$, the surface pressure spectra displays a hump centered at $f c / U=1.4$. This hump then decreases until the spectrum reaches a power law when the flow is fully stalled. For very high angles of attack $\left(\alpha_{s, g}>27^{\circ}\right)$, similar surface pressure spectra are observed for both airfoils, with a narrow band peak centered at $S t=f c \sin \left(\alpha_{s, g}\right) / U \simeq 0.19$. This peak can be explained by shear layer instabilities and large scale periodic vortex shedding [3].

PSD of the far-field acoustic pressure are presented in Fig. 5 for the same angles of attack. For low angles of attack, the signal to noise ratio is low, with background noise dominating for $f c / U<1$ and $f c / U>10$. Between these frequencies, comparable spectra are obtained for both airfoils. For the NACA0012, the abrupt stall at $\alpha_{s, g}=15^{\circ}$ leads 


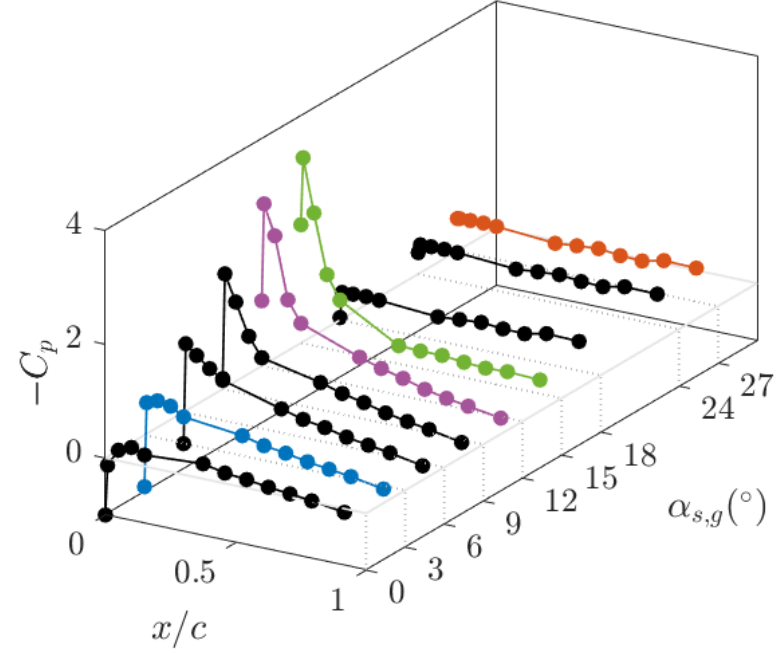

(a)

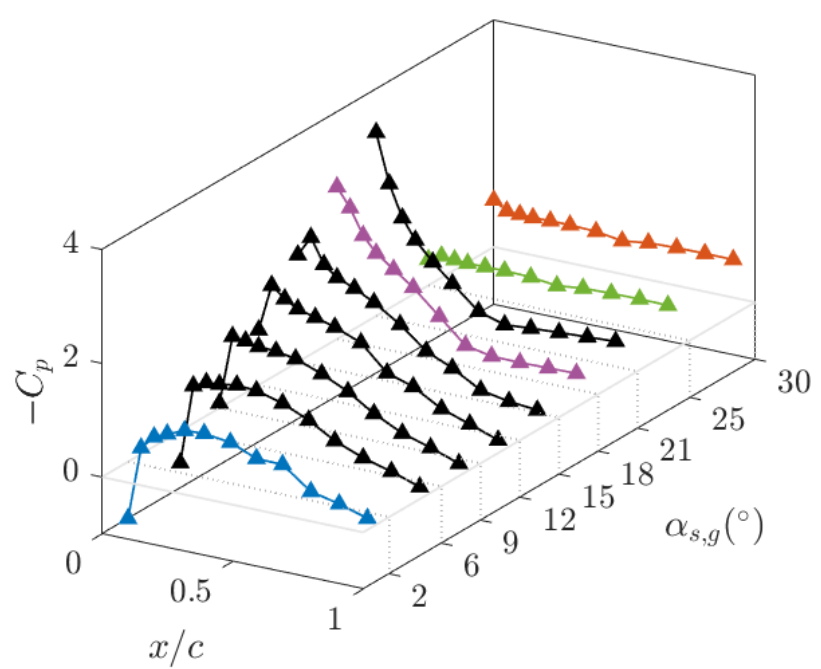

(b)

Fig. 3 Distribution of static pressure on the suction side of (a) the NACA0012 and (b) the NACA63 418 at various angles of attack. The colors correspond to the colors from Fig. 4 and 5 .

to a rapid increase of the spectra amplitude at low frequencies, with an increase of the noise amplitude up to $15 \mathrm{~dB}$ for $f c / U=1$ compared to the pre-stall regime. This broadband noise corresponds to the so called "light-stall" regime. For the NACA $63_{3} 418$, the transition to stall noise is smoother, with the spectra amplitude progressively increasing and shifting to lower frequencies as the separation point gets closer to the leading edge. The abrupt stall at $\alpha_{s, g}=25^{\circ}$ leads the spectra to increase at low frequencies but to decrease for $0.7<f c / U<2.5$. Note that this light-stall regime is less noisy than the one for the NACA0012. For very high angles of attack $\left(\alpha_{s, g}>27^{\circ}\right)$, similar deep-stall regimes are obtained for both airfoils, with a narrow-band peak at $S t=f c \sin \left(\alpha_{s, g}\right) / U \simeq 0.19$. The two airfoils thus exhibit similar bulk body behaviors at very high angles of attack. Similar observation was made by Laratro et al. [4] for a NACA0012 and a NACA0021 at very high angles of attack. 


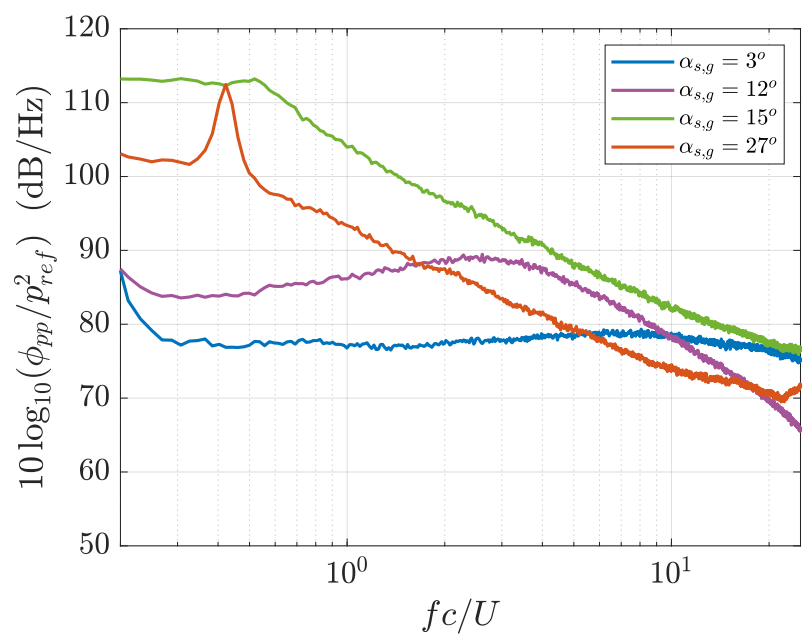

(a)

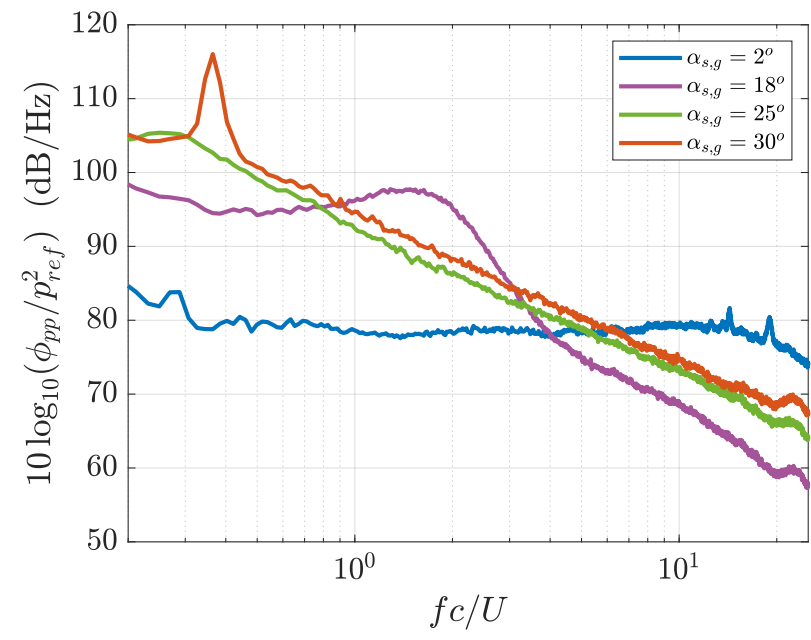

(b)

Fig. 4 Surface pressure PSD at $x / c=0.92$ on the suction side for various static angles of attack and for (a) the NACA0012 and (b) the NACA63 418.

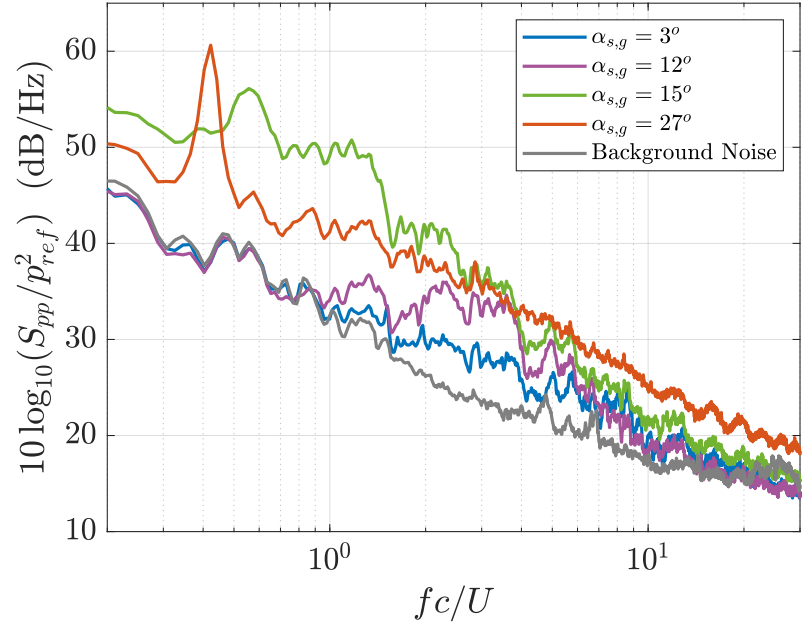

(a)

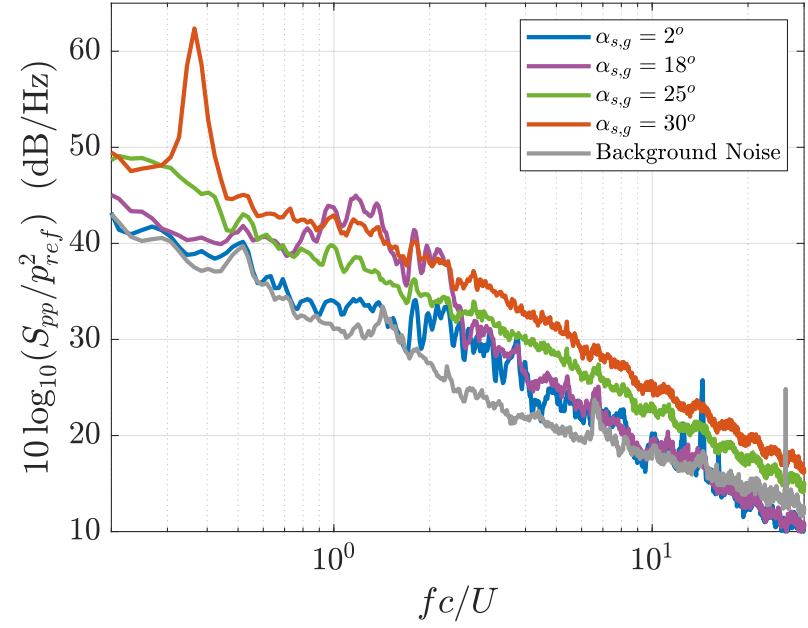

(b)

Fig. 5 PSD of far-field acoustic pressure for various static angles of attack and for (a) the NACA0012 and (b) the NACA63 3418 . 

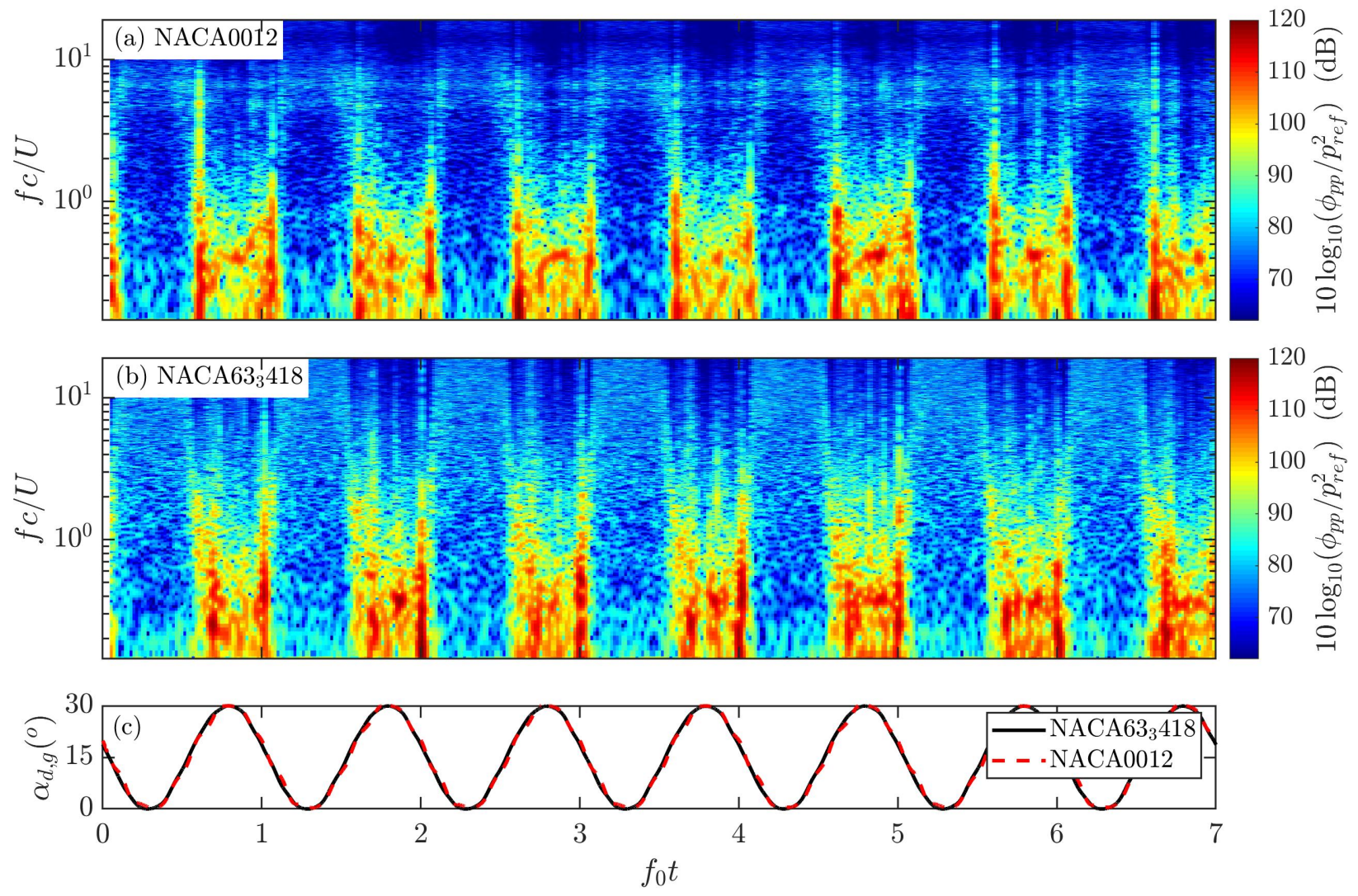

Fig. 6 Instantaneous spectrograms of surface pressure at $x / c=0.92$ on the suction side for (a) the NACA0012 and (b) the NACA63 418 , and (c) instantaneous angle of attack for $\alpha_{d, g}=15^{\circ}+15^{\circ} \sin (2 \pi f t)$ and $k=0.01$.

\section{Oscillating airfoil}

\section{A. Time-frequency analysis}

A similar investigation is now presented for the oscillating airfoil cases. Far-field noise and surface pressure are measured for 100 oscillation periods. Spectrograms are then computed with $80 \%$ overlap and window sizes of 7000 samples, 3500 samples and 1400 samples for $f_{0}=0.66 \mathrm{~Hz}, f_{0}=1.33 \mathrm{~Hz}$ and $f_{0}=3.32 \mathrm{~Hz}$, respectively, in order to have the same number of windows per cycle. Segments are extended to 8192 samples using zero-padding to obtain the same frequency resolution for every oscillation frequency. The resulting spectrograms have a frequency resolution of $6.25 \mathrm{~Hz}$, and time resolutions of $27 \mathrm{~ms}, 14 \mathrm{~ms}$ and $6 \mathrm{~ms}$, respectively.

Spectrograms of surface pressure at $x / c=0.92$ on the suction side of the airfoils and spectrograms of far-field acoustic pressure are presented in Fig. 6 and 7 for $k=0.01$. For both airfoils, periodic variations of the spectral amplitude are observed. Each time the angle of attack increases above a critical value, an increase of the spectra is observed at low frequencies. The amplitudes of the spectra remain high up until the angle of attack decreases below a value low enough so that the flow can reattach on the suction side. High angles of attack are also associated with a decrease of the surface pressure spectra for $f c / U>10$, in good agreement with Mayer et al. [10]. Similarities between the surface pressure and far-field pressure spectrograms show that the boundary layer is constantly adapting to the angle of attack of the airfoil, leading the sound generation mechanisms to be modified throughout the oscillation. No clear effect of the airfoil shape is visible.

Phase-averaged spectrograms of surface pressure near the trailing-edge are presented in Fig. 8 and phase-averaged spectrograms of far-field acoustic pressure are presented in Fig. 9. To avoid transient effects, the first 10 oscillation periods are discarded from the analysis, and phase-averaging of the spectrograms is performed with respect to the angle of attack on the 90 remaining cycles. Similarly to the static case, the spectrograms exhibit two distinct regimes during 

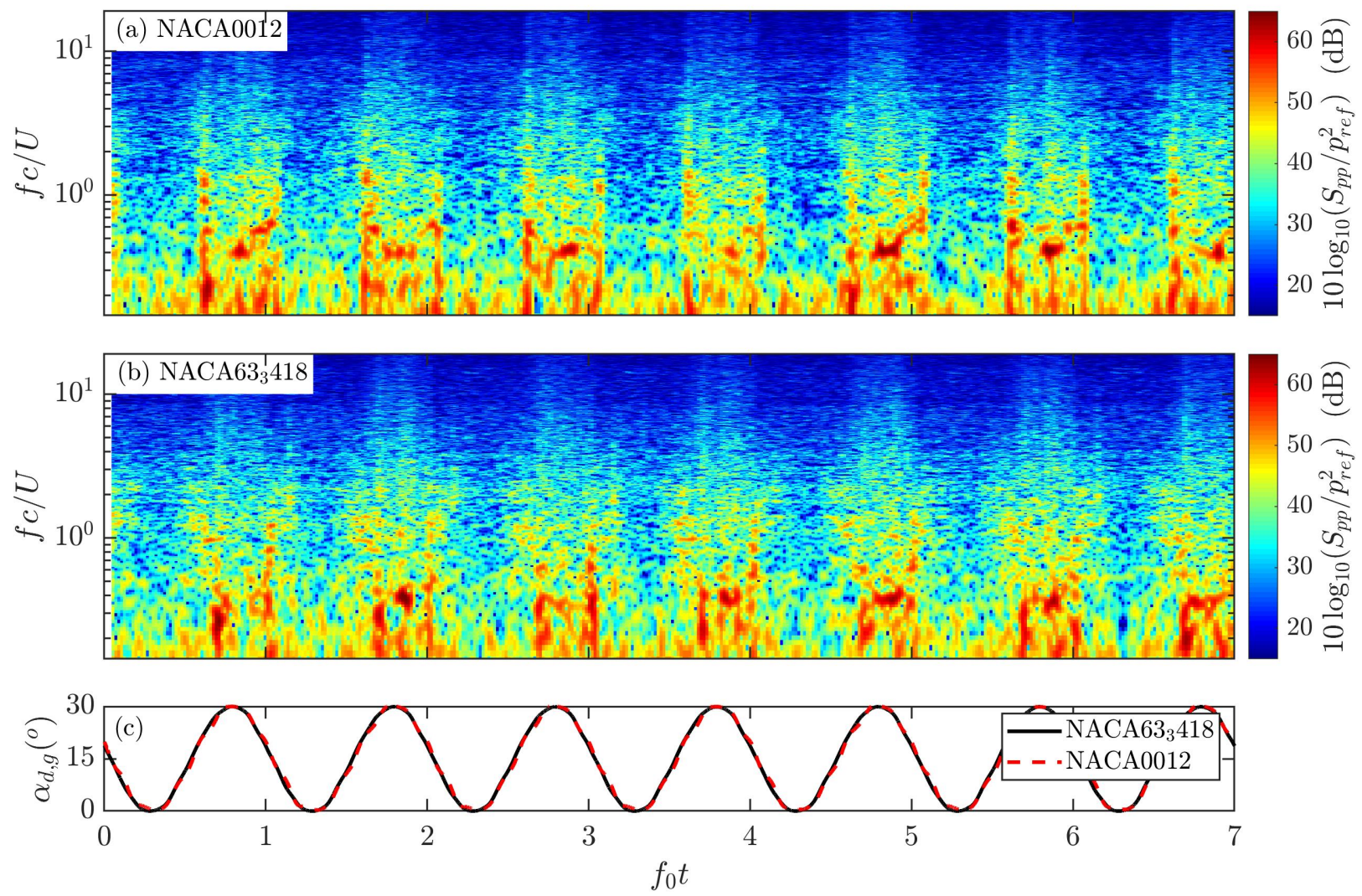

Fig. 7 Instantaneous spectrograms of far-field acoustic pressure for (a) the NACA0012 and (b) the NACA63 418 , and (c) instantaneous angle of attack for $\alpha_{d, g}=15^{\circ}+15^{\circ} \sin (2 \pi f t)$ and $k=0.01$. 

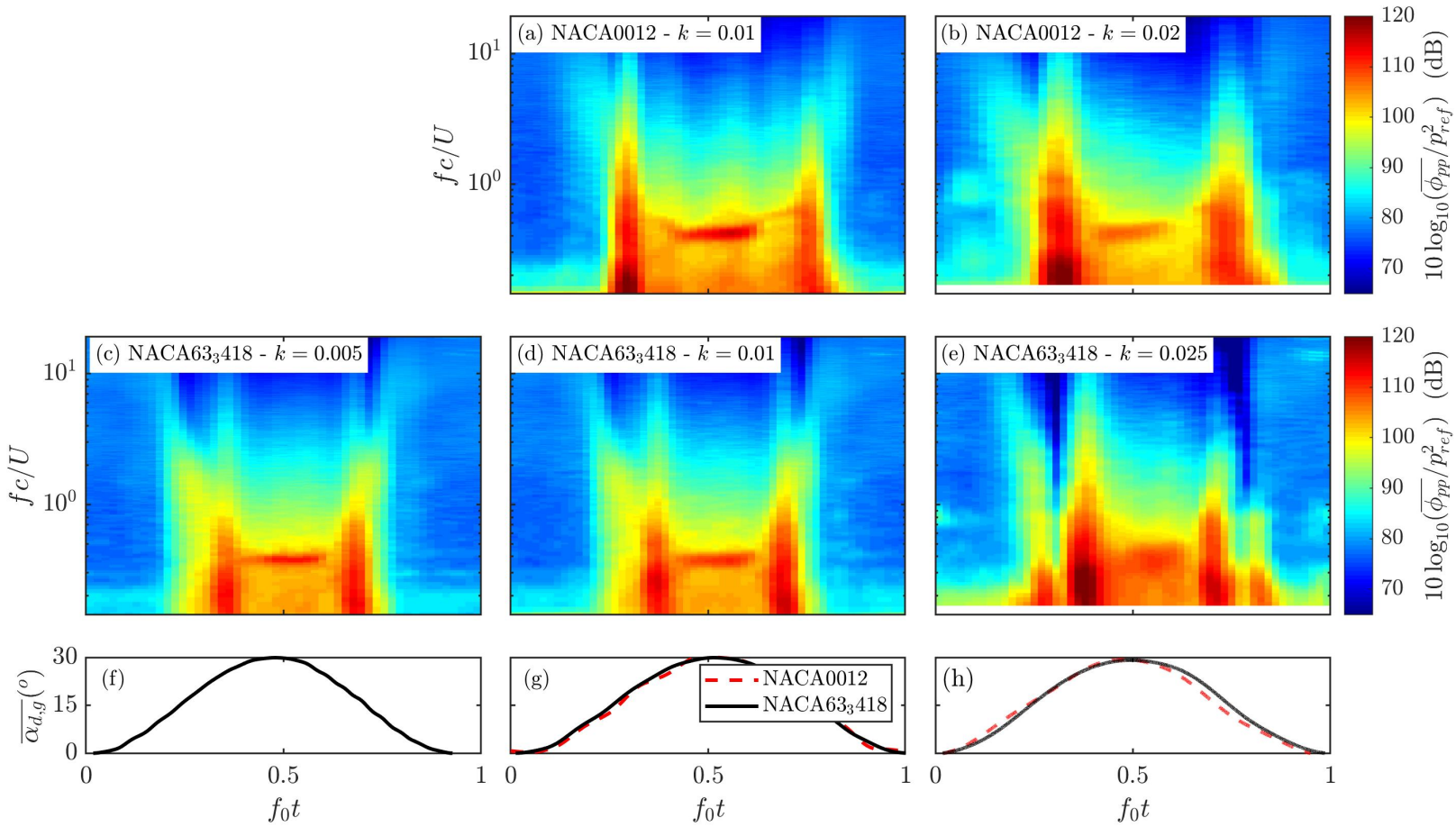

Fig. 8 Phase-averaged spectrograms of surface pressure at $x / c=0.92$ on the suction side for $\alpha_{d, g}=15^{\circ}+$ $15^{\circ} \sin \left(2 \pi f_{0} t\right)$ for (a-b) the NACA0012 airfoil and (c-d-e) the NACA63 418 airfoil, and (f-g-h) phase-averaged angle of attack. The overbar denotes phase-averaging.

the stalled phase of the cycle. At the stall onset and near flow reattachment, a low frequency broadband spectrum is obtained. In between these two instants, the energy gathers in a narrow-band peak, the center frequency of which varies with time as the angle of attack continues its sinusoidal motion, so that the peak is continuously located at a Strouhal number $S t \simeq 0.2$ with $S t=f c \sin \left(\overline{\alpha_{d, g}}\right) / U$ and $\overline{\alpha_{d, g}}$ the phase-averaged angle of attack. The light-stall and deep-stall regimes commonly observed for static airfoils are thus also present for oscillating airfoils. In the same way as for the static configurations, the light-stall and deep-stall regimes are also observed on the surface pressure spectrograms, with the stall onset and flow reattachment diplaying high amplitude broadband behaviors. Note that a pollution is present on the far-field acoustic pressure spectrogram for the NACA63 418 at $k=0.025$, as shown with the dashed line in Fig. 9 . The most probable source of this pollution is the motor under the lower end plate. This contamination only happens during the downstroke motion of the airfoil and is assumed not to have an effect on the rest of the spectrogram.

For both airfoils, a similar effect of the reduced frequency on the dynamic stall noise is observed. For $k=0.01$, the far-field pressure spectrograms are nearly symmetrical and the broadband noises at the stall onset and near flow reattachment are similar. For $k>0.02$, the spectrograms do not exhibit a symmetrical behavior between stall onset and flow reattachment. At the stall onset, the amplitude and duration of the broadband noise increase, whereas the broadband noise close to flow reattachment has similar amplitude and duration than the one for $k=0.01$. Similar effect is observed on the surface pressure spectra. Increasing the frequency of the oscillation thus leads to an increase of the pressure fluctuations amplitude near the trailing-edge at the dynamic stall onset. This results agrees with previous findings at lower Reynolds number [11]. The deep-stall noise is also impacted by the increase of the reduced frequency, with an increase of the narrow-band peak width for higher reduced frequency (see Fig. 9 $\mathrm{d}$ and $9 \mathrm{p}$ ). This could originate from the large scale vortex shedding being less coherent at high reduced frequency, as its frequency has to constantly adapt to the rapidly changing angle of attack.

Effects of the airfoil shape are visible in Fig. 9. Firstly, the stalled phase of the cycle is shorter for the cambered airfoil than for the symmetrical one. For $k=0.01$, the stall onset broadband noise takes place at $f_{0} t \sim 0.28$ for the NACA0012 and at $f_{0} t \sim 0.36$ for the NACA63 418 (see Fig. 9a and 9d). Moreover, this broadband noise is limited to frequencies below $f c / U<1$ for the NACA63 3418 and spreads up to $f c / U=2$ for the symmetrical airfoil. In the same 

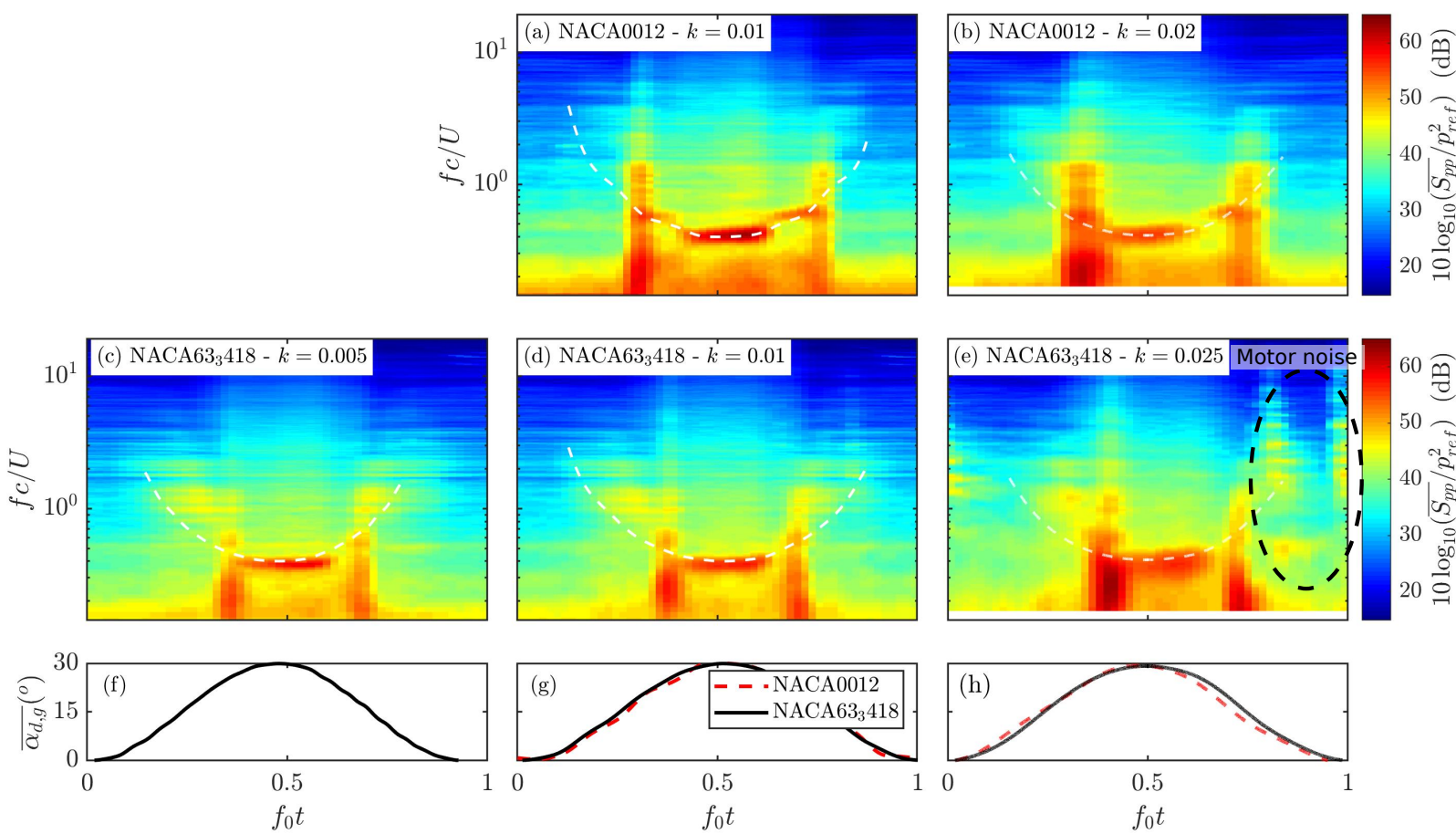

Fig. 9 Phase-averaged spectrograms of far-field acoustic pressure for $\alpha_{d, g}=15^{\circ}+15^{\circ} \sin \left(2 \pi f_{0} t\right)$ for (a-b) the NACA0012 airfoil and (c-d-e) the NACA63 418 airfoil, and (f-g-h) phase-averaged angle of attack. The overbar denotes phase-averaging. The dashed white line shows the frequency corresponding to a constant Strouhal number $S t=f c \sin \left(\overline{\alpha_{d, g}}\right) / U$ with $\overline{\alpha_{d, g}}$ the phase-averaged angle of attack. The black dashed ellipse shows the contamination by the motor noise.

way as for the static regime, the stall noise of the NACA63 3418 is preceded by an increase of the far-field acoustic spectra amplitude centered at $f c / U \simeq 1$ due to the flow gradually separating from the suction of the airfoil. A gradual broadband increase of the surface pressure spectra near the trailing edge before the dynamic stall onset is also observed (see Fig. 8d). This separation noise is not observed for the NACA0012 airfoil. Finally, the center frequency of the narrow-band peak varies much less in the case of the cambered airfoil. This is due to a much reduced range of angles of attack visited during the deep-stall phase of the cycle compared to the NACA0012, leading to a reduced range of variation of the vortex shedding frequency.

\section{B. Overall Sound Pressure Level}

The Overall Sound Pressure Level (OASPL) is defined as:

$$
\text { OASPL }=10 \log _{10}\left(\frac{1}{p_{\text {ref }}^{2}} \int_{f_{1}}^{f_{2}} S_{p p} d f\right),
$$

with $p_{\text {ref }}=20 \mu \mathrm{Pa}$ the reference pressure and $f_{1}=70 \mathrm{~Hz}$ and $f_{2}=1000 \mathrm{~Hz}$. These values are chosen in order to capture the noise amplitude variations in the stall noise frequency range. The phase-averaged OASPL during the pitching motion is compared to the phase-averaged lift coefficient $\overline{C_{l}}$ in Fig. 10 for the NACA63 3418 and various reduced frequencies. For every reduced frequencies, the OASPL is progressively increasing before the onset of stall. Maxima of OASPL are then observed at the stall onset and near flow reattachment, corresponding to the broadband noises obtained near the critical stall and reattachment angles. In between these instants, a decrease of the OASPL is observed, corresponding to the dynamic deep-stall noise regime. For $k=0.005$ and $k=0.01$, similar OASPL curves are obtained. However for $k=0.025$, an increase of the width and maximum value of the stall onset OASPL peak is observed. The peak reaches OASPL $=78 \mathrm{~dB}$ for $k=0.005$ and OASPL $=83 \mathrm{~dB}$ for $k=0.025$. Moreover, increasing the reduced frequency 

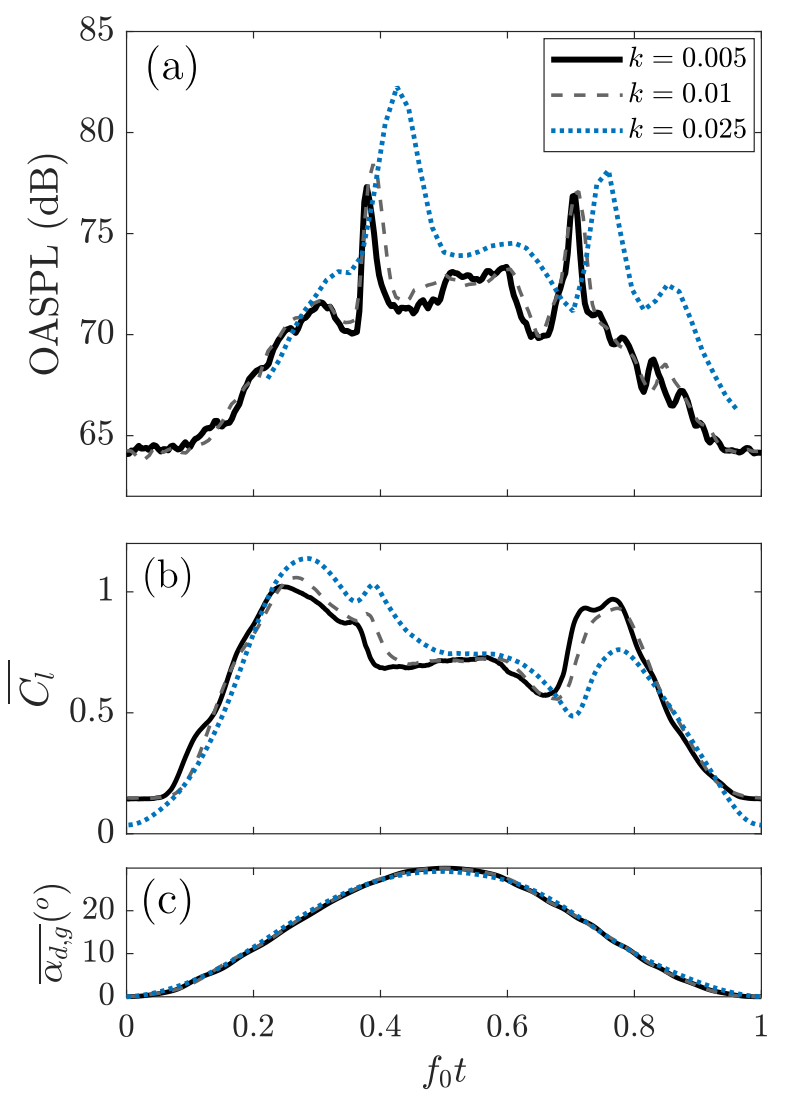

Fig. 10 Phase-averaged (a) OASPL, (b) lift coefficient and (c) angle of attack during the dynamic stall phenomenon for the $\mathrm{NACA63}_{3} 418$ airfoil at various reduced frequencies. For the cambered airfoil and $k=0.025$, the OASPL is cut for angles where the motor noise is dominating.

leads to a delay of the maximum of the peak, the maximum taking place at $f_{0} t=0.38$ for $k=0.005$ and $f_{0} t=0.43$ for $k=0.025$. This delay of stall is also visible on the phase-averaged lift coefficient. Similarities between results for $k=0.005$ and $k=0.01$ could indicate that these regimes are quasisteady, and that unsteady effects of the dynamic stall are negligible under a reduced frequency threshold.

Effects of the airfoil shape on the OASPL are investigated in Fig. 11 for $k=0.01$. For the NACA $63_{3} 418$, the stall onset OASPL peak appears to have lower amplitude and be delayed to larger angles of attack compared to the NACA0012. In the same way, the OASPL peak at flow reattachment takes place earlier for the NACA $63_{3} 418$ than for the NACA0012, but the peaks have the same amplitude. The duration of the stalled phase of the cycle is thus shorter for the cambered airfoil. The OASPL hump centered at $f_{0} t \sim 0.58$ corresponding to the deep-stall regime also has a lower amplitude for the cambered airfoil. For the NACA0012, the OASPL is flat before stall and after flow reattachment $\left(f_{0} t<0.2\right.$ and $\left.f_{0} t>0.8\right)$ and stall leads to an abrupt increase of OASPL, from OASPL $=67 \mathrm{~dB}$ at $f_{0} t=0.24$ to OASPL $=82 \mathrm{~dB}$ at $f_{0} t=0.31$. For the NACA63 418 , a progressive increase of OASPL is observed before stall onset due to the gradual flow separation on the suction side of the airfoil, leading this airfoil to be the noisiest before the stall onset and after flow reattachment.

\section{Conclusion and future work}

Combined far-field acoustic and surface pressure measurements were performed in an anechoic open-jet wind-tunnel in order to study the effect of the airfoil shape on the static and dynamic stall noises. For static configurations, the NACA63 3418 airfoil offers a smoother transition to the stall noise, with a gradual increase of the spectral amplitude at low frequencies, as the separation moves from the trailing edge to the leading edge. After the abrupt stall, the light-stall 


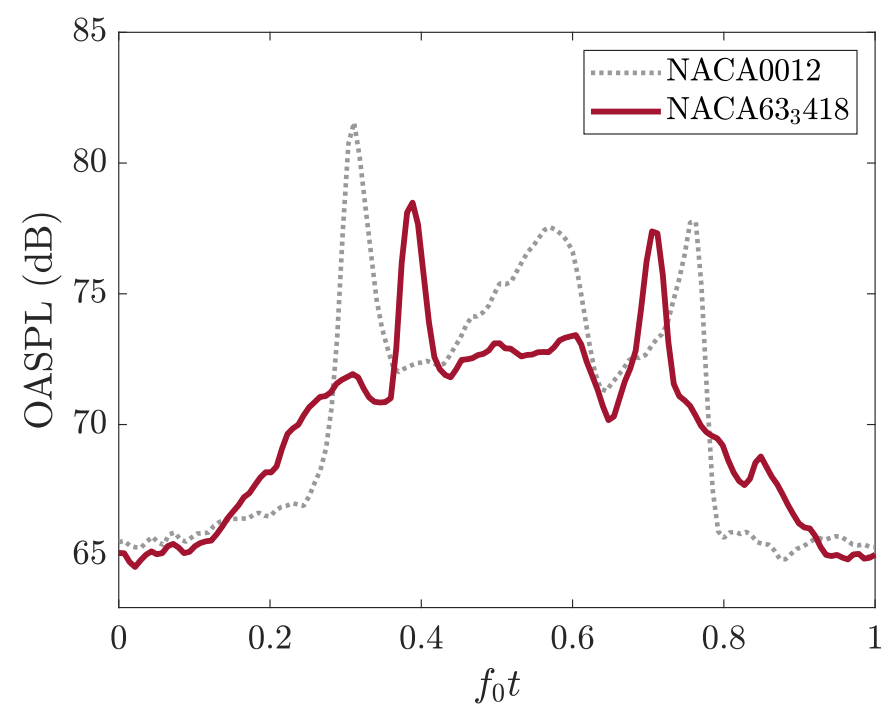

Fig. 11 Phase-averaged OASPL during the dynamic stall phenomenon for the two airfoils and $k=0.01$.

noise has a lower amplitude for this airfoil than for the NACA0012 airfoil. At very high angle of attack, no effect of the airfoil shape is observed. Both airfoils exhibit a similar bulk body behavior, with the presence of a narrow-band peak at Strouhal number $S t=0.19$ dominating the far-field noise, and a decrease of the broadband component of the noise.

In the same way as for the static stall noise, the dynamic stall noise is characterized by an increase of the amplitude of the noise at low frequencies when the angle of attack increases above a critical angle, corresponding to the dynamic stall angle. The amplitude of the noise remains high during the stalled phase of the oscillation, and then decreases as the angle of attack becomes low enough for the flow to reattach on the suction side of the airfoil. The light-stall and deep-stall noise regimes observed for static airfoils are also taking place for the oscillating airfoils.

Effects of the reduced frequency and the airfoil shape on the dynamic stall noise were investigated. Stall onset broadband noise is delayed to larger angles of attack as the reduced frequency increases. This result agrees with previous findings at lower Reynolds number [11] and with the commonly observed boundary layer separation delay obtained in dynamic stall experiments [18]. Increasing the reduced frequency of the oscillation also leads to an increase of the amplitude and duration of the stall onset broadband noise. This phenomenon is not explained yet and could originate from the complex flow structures developing during the dynamic stall process. Finally, the deep-stall narrow-band peak is widening as the reduced frequency increases. This could be explained by the fact that the large scale vortex shedding becomes less coherent for rapidly changing incidence as the shedding frequency fails to adapt to the instantaneous angle of attack of the airfoil. In the future, synchronized acoustic and time-resolved Particle Image Velocimetry (PIV) measurements will be conducted, in order to understand the role of the flow structures around the airfoil on the sound generation mechanisms. Moreover, unsteady surface pressure measurements on the suction side of the airfoil will be analyzed in order to estimate the spanwise correlation length and convection velocity near the trailing edge during the oscillation. These measurements will provide a better insight on the turbulent structures developing during the pitching motion and responsible for the far-field noise.

With the NACA63 3418 airfoil, the dynamic stall noise has a lower amplitude and is delayed to larger angles of attack compared to the experiments with the NACA0012 airfoil. Not only this airfoil shape has a good aerodynamic efficiency, but it is also good to limit dynamic stall noise and strong amplitude modulations of the wind turbine noise. However, the NACA63 3418 airfoil is noisier in the pre-stall phase of the cycle, with the presence of a separation noise hump centered at $f c / U \sim 1$, while the noise emitted by the NACA0012 airfoil does not depend on the angle of attack below stall. For oscillations below the stall angle, using the NACA63 418 airfoil could thus increase the amplitude modulation of the wind turbine noise compared to a thinner and symmetrical airfoil. Further investigations are thus needed in order to segregate the effects of camber and thickness on the separation noise. 


\section{Acknowledgments}

This research is funded by the French National Agency for Research (ANR) under grant agreement $\mathrm{N}^{\circ}$ ANR-18CE04-0011 and by the Agence Innovation Défense under grant agreement $N^{\circ} 2018600071004707501$. It was performed within the framework of the LABEX CelyA (ANR-10-LABX-0060) of Universite de Lyon, within the program Investissements d'Avenir (ANR-16-IDEX-0005) operated by the ANR.

\section{References}

[1] Oerlemans, S., "Effect of Wind Shear on Amplitude Modulation of Wind Turbine Noise," International Journal of Aeroacoustics, Vol. 14, No. 5-6, 2015, pp. 715-728.

[2] Brooks, T. F., Pope, D., and Marcolini, M., “Airfoil self-noise and prediction,” NASA Reference Publication $1218,1989$.

[3] Moreau, S., Roger, M., and Christophe, J., "Flow Features and Self-Noise of Airfoils Near Stall or in Stall," 15th AIAA/CEAS Aeroacoustics Conference (30th AIAA Aeroacoustics Conference), 2009.

[4] Laratro, A., Arjomandi, M., Cazzolato, B., and Kelso, R., "Self-noise and directivity of simple airfoils during stall: An experimental comparison,” Applied Acoustics, Vol. 127, 2017, pp. 133 - 146.

[5] Bertagnolio, F., Madsen, H., Fischer, A., and Bak, C., "A semi-empirical airfoil stall noise model based on surface pressure measurements," Journal of Sound and Vibration, Vol. 387, 2016.

[6] Lacagnina, G., Chaitanya, P., Berk, T., Kim, J.-H., Joseph, P., Ganapathisubramani, B., Hasheminejad, S. M., Chong, T. P., Stalnov, O., Choi, K.-S., Shahab, M. F., Omidyeganeh, M., and Pinelli, A., "Mechanisms of airfoil noise near stall conditions," Phys. Rev. Fluids, Vol. 4, 2019, p. 123902.

[7] Turner, J., and Kim, J., "Aerofoil dipole noise due to flow separation and stall at a low Reynolds number," International Journal of Heat and Fluid Flow, Vol. 86, 2020, p. 108715.

[8] Siegel, L., Ehrenfried, K., Wagner, C., Mulleners, K., and Henning, A., "Cross-correlation analysis of synchronized PIV and microphone measurements of an oscillating airfoil," Journal of Visualization, Vol. 21, No. 3, 2018, pp. 381-395.

[9] Zhou, T., Sun, Y., Fattah, R., Zhang, X., and Huang, X., "An experimental study of trailing edge noise from a pitching airfoil," The Journal of the Acoustical Society of America, Vol. 145, No. 4, 2019, pp. 2009-2021.

[10] Mayer, Y., Zang, B., and Azarpeyvand, M., "Aeroacoustic investigation of an oscillating airfoil in the pre- and post-stall regime," Aerospace Science and Technology, Vol. 103, 2020, p. 105880.

[11] Raus, D., Cotté, B., Monchaux, R., Jondeau, E., Souchotte, P., and Roger, M., "Experimental study of the dynamic stall noise on an oscillating airfoil," Manuscript submitted for publication, .

[12] Abbott, I. H., and Von Doenhoff, A. E., Theory of wing sections: including a summary of airfoil data, Courier Corporation, 1959.

[13] Troldborg, N., Bak, C., Madsen, H. A., and Skrzypinski, W. R., "Danaero mw," 2013.

[14] Grasso, F., "Usage of numerical optimization in wind turbine airfoil design," Journal of Aircraft, Vol. 48, No. 1, 2011 , pp. $248-255$.

[15] Brooks, T. F., Marcolini, M., and Pope, D., "Airfoil trailing edge flow measurements and comparison with theory, incorporating open wind tunnel corrections," 9th Aeroacoustics Conference, 1984.

[16] Sheng, W., Galbraith, R. A. M., and Coton, F. N., "Prediction of Dynamic Stall Onset for Oscillatory Low-Speed Airfoils," Journal of Fluids Engineering, Vol. 130, No. 10, 2008.

[17] Roger, M., "Microphone measurements in aeroacoustic installations," Design and Operation of Aeroacoustic Wind Tunnel Tests for Ground and Air Transport STO-AVT-287. Von Karman Institute for Fluid Dynamics, 2017.

[18] Lee, T., and Gerontakos, P., "Investigation of flow over an oscillating airfoil," Journal of Fluid Mechanics, Vol. 512, 2004, pp. 313-341. 